\title{
2-amino-1,3-benzothiazole-6-carboxamide Preferentially Binds the Tandem Mismatch Motif r(UY:GA)
}

Andrew T. Chang ${ }^{1,2 \#}$, Lu Chen ${ }^{3,4 \#}$, Luo Song ${ }^{4 \#}$, Shuxing Zhang ${ }^{4}$ and Edward P. Nikonowicz ${ }^{1 *}$

${ }^{1}$ Department of BioSciences, Rice University, Houston, TX 77251-1892

2Department of Medicine, Division of Endocrinology, Gerontology, and Metabolism, Stanford Medicine, Stanford, CA 94305-5103

${ }^{3}$ Department Division of Preclinical Innovation, National Institutes of Health, Bethesda, MD 20892-0001

${ }^{4}$ Intelligent Molecular Discovery Laboratory, Department of Experimental Therapeutics, MD Anderson Cancer Center, Houston, TX 77054

This work was supported by National Science Foundation grant CHE-1412864 and CHE-1411859 to E.P.N. and S.Z., respectively.

\#Contribute equally

*Corresponding authors.

edn@rice.edu and shuzhang@mdanderson.org

Key Words: RNA hairpin, RNA motif, small molecule, ensemble docking, molecular dynamics simulation 


\section{Abstract}

RNA helices are often punctuated with non-Watson-Crick features that can be the target of chemical compounds, but progress towards identifying small molecules specific for non-canonical elements has been slow. We have used a tandem UU:GA mismatch motif (5'-UG-3':5'-AU-3') embedded within the helix of an RNA hairpin as a model to identify compounds that bind the motif specifically. The three-dimensional structure of the RNA hairpin and its interaction with a small molecule compound identified through a virtual screen are presented. The G-A of the mismatch forms a sheared pair upon which the $U-U$ base pair stacks. The hydrogen bond configuration of the $\mathrm{U}-\mathrm{U}$ pair involves the $\mathrm{O} 2$ of the $\mathrm{U}$ adjacent to the $\mathrm{G}$ and the $\mathrm{O}$ 4 of the $\mathrm{U}$ adjacent to the A. The G-A and $U-U$ pairs are flanked by $A-U$ and $G-C$ base pairs, respectively, and the mismatch exhibits greater stability than when the motif is within the context of other flanking base pairs or when the 5'-3' orientation of the G-A and U-U is swapped. Residual dipolar coupling constants were used to generate an ensemble of structures against which a virtual screen of 64,480 small molecules was performed to identify candidate compounds that the motif specifically binds. The tandem mismatch was found to be specific for one compound, 2-amino-1,3-benzothiazole-6-carboxamide, which binds with moderate affinity but extends the motif to include the flanking $A-U$ and $G-C$ base pairs. The finding that affinity for the UU:GA mismatch is flanking sequence dependent emphasizes the importance of motif context and potentially increases the number of small non-canonical features within RNA that can be specifically targeted by small molecules. 


\section{Introduction}

Non-coding RNA (ncRNA) molecules including microRNAs (aka, miRNAs) span a wide range of roles in biology including functioning as metabolic sensors, as scaffolds for assembly of large ribonucleoprotein complexes, and as direct suppressors of gene expression. To engage in their myriad functions, these molecules utilize both nucleotide sequence and shape (secondary and tertiary structure). Structured RNA molecules are primarily composed double helices that may contain conserved small structural motifs such as K-turns, adenine platforms, and loop E motifs. For RNAs with multiple helices, the arrangement of helices can be stabilized by tertiary interactions that include coaxial stacking, tetraloop:tetraloop-receptor interactions, and ribose zippers. RNA helices themselves are often interrupted by non-canonical elements such as non-Watson-Crick base pairs, bulged nucleotides, and internal loops. These elements, that can be central to an RNA molecule's function, punctuate the regular A-form geometry of the helix with distinctive structural features or introduce local dynamic characteristics within an otherwise well-ordered helix.

Owing to their numerous cellular functions and their propensity to form secondary (and tertiary) structure, ncRNAs are attractive for their potential as targets of small therapeutic compounds ${ }^{4,5}$. Additionally, chemical compounds that are selective for specific structural features presented by an RNA molecule also can be versatile tools for RNA structure analysis and functional studies. The features recognized by many antibiotics that bind rRNA include regular helices and non-canonical elements and the same types of features are present in all RNAs. Further, multiple occurrences of bulged nucleotides and internal loops within a helix can establish a structural and dynamics 
pattern unique to the RNA molecule containing those features. Although non-canonical elements within regular helices offer clear opportunities for targeting specific ncRNAs, progress towards the identification of small molecules that selectively bind such nonstandard features has only recently begun to gain momentum ${ }^{6-10}$.

One lesser common irregular secondary structure element is the tandem UU:GA base pair mismatch. This mismatch motif occurs in the helices of large rRNA subunits of archaea, in many eukaryotic rRNA subunits in the region homologous to the bacterial ribosomal protein L11 binding site, within an HIV-1 intronic splicing silencer (ISS) stemloop ${ }^{11}$, and within the intermolecular helix formed between the anticodon arm of human tRNA ${ }^{\text {Lys3 }}$ and the HIV A-rich Loop I adjacent to the HIV primer binding site ${ }^{12}$. The UU:GA motif also forms part of the binding site of an aptamer of ribosomal protein S8 ${ }^{13}$ and has been observed in the stems of at least 24 immature human miRNA molecules including miR-382 ${ }^{14}$, miR-496 ${ }^{15}$, and miR-508 ${ }^{16}$. The tandem mismatch within the aptamer facilitates formation of a tertiary structure necessary for protein binding and may play a similar role in the rRNA L11 protein binding site ${ }^{17}$. Structural studies of RNAs containing the UU:GA mismatch also suggest that the identity of the flanking base pairs may influence the structure and dynamics of the mismatch ${ }^{11-13,17}$.

We have used heteronuclear NMR spectroscopy to study the solution structure of a tandem UU:GA mismatch motif within an RNA helix and have identified a small molecule, ZN423 (IUPAC name: 2-amino-1,3-benzothiazole-6-carboxamide), that binds this motif. The mismatched nucleotides form $U-U$ and sheared G-A base pairs with $U_{7}$ $\mathrm{O} 2$ and $\mathrm{U}_{22} \mathrm{O} 4$ serving as the hydrogen bond acceptors within the $\mathrm{U}-\mathrm{U}$ base pair. ZN423 broadens the base resonances that flank the G-A pair that reflect chemical 
exchange on the intermediate timescale. Although the binding affinity of ZN423 to the RNA is moderate $\left(K_{D}>100 \mathrm{uM}\right)$, ZN423 exhibits selectivity for the motif. In addition, binding of ZN423 was found to be dependent on the identity of the base pairs flanking the UU:GA motif. Finally, although substitution of $U_{7}$ with cytidine supports $Z N 423$ binding, the $\mathrm{U}_{7}-\mathrm{C}_{22}$ variant does not bind ZN423. A structural model of the ZN423-RNA complex is presented.

\section{Materials and methods}

All enzymes were purchased (Sigma) with the exception of T7 RNA polymerase which was prepared as described ${ }^{18}$. Deoxyribonuclease I Type II, pyruvate kinase, adenylate kinase, and nucleotide monophosphate kinase were obtained as powders and dissolved into $15 \%$ glycerol, $1 \mathrm{mM}$ dithiothreitol and $10 \mathrm{mM}$ Tris- $\mathrm{HCl}, \mathrm{pH} 7.4$ and stored at $-20{ }^{\circ} \mathrm{C}$. Guanylate kinase and nuclease P1 were obtained as solutions and stored at $-20{ }^{\circ} \mathrm{C}$.

Unlabeled 5' nucleoside triphosphates (Sigma), phosphoenolpyruvate (potassium salt)

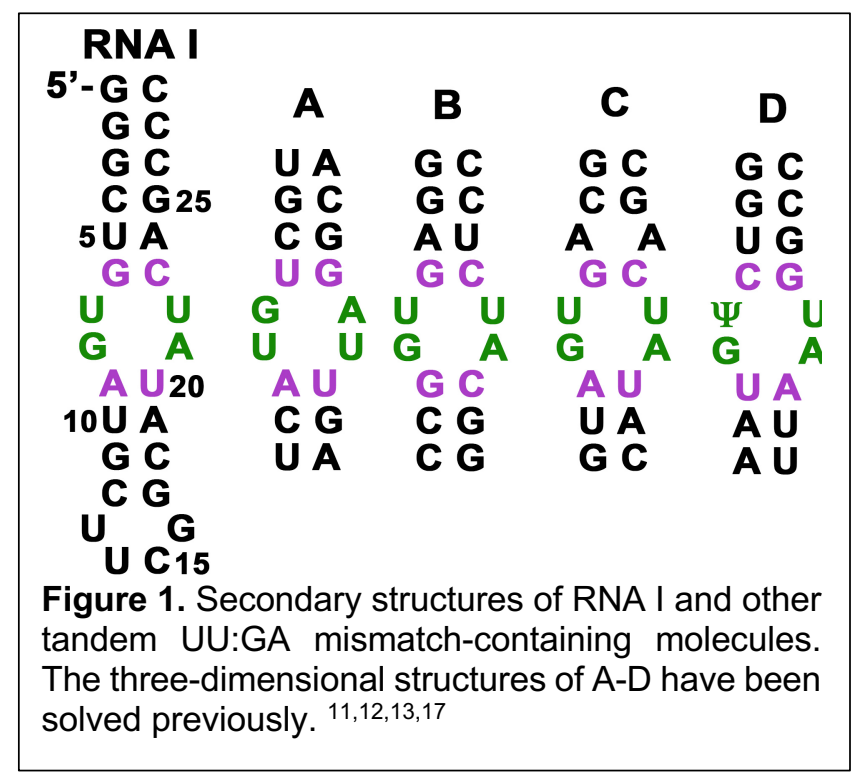

(Bachem), and 99\% $\left[{ }^{15} \mathrm{~N}\right]$ ammonium sulfate and $99 \%\left[{ }^{13} \mathrm{C}_{6}\right]$-glucose (Cambridge Isotope Labs) were obtained as powders. The identified hit compounds were purchased from ChemBridge Corporation. Preparation of RNA Samples. RNA I, (Figure 1), was prepared by in vitro transcription using T7 RNA polymerase and a synthetic DNA template ${ }^{19}$. Unlabeled 
RNA molecules were prepared from $10 \mathrm{ml}$ transcription reactions using 4 mM 5'-NTPs. Isotopically enriched RNA molecules were prepared from $6 \mathrm{ml}$ transcription reactions using $3 \mathrm{mM}{ }^{13} \mathrm{C} /{ }^{15} \mathrm{~N}$-enriched $5^{\prime}-\mathrm{NTPs}$ as described ${ }^{20}$. The RNA molecules were purified (20\% w/v preparative PAGE), electroeluted (Schleicher \& Schuell), and ethanol precipitated. The RNA was dissolved in $1.0 \mathrm{M} \mathrm{NaCl}, 20 \mathrm{mM}$ potassium phosphate, $\mathrm{pH}$ 6.8, and 2.0 mM EDTA and dialyzed extensively against $10 \mathrm{mM} \mathrm{NaCl}, 5 \mathrm{mM}$ potassium phosphate, pH 6.8, and 0.05 mM EDTA using a Centricon-3 concentrator (Amicon Inc.). The samples were diluted with buffer to a volume of $0.3 \mathrm{ml}$ and lyophilized to powders. For experiments involving the non-exchangeable protons, the samples were exchanged twice from $99.9 \% \mathrm{D}_{2} \mathrm{O}$ and dissolved in $0.33 \mathrm{ml}$ of $99.96 \% \mathrm{D}_{2} \mathrm{O}$. For experiments involving the exchangeable protons, the samples were dissolved in $0.33 \mathrm{ml}$ of $90 \%$ $\mathrm{H}_{2} \mathrm{O} / 10 \% \mathrm{D}_{2} \mathrm{O}$. The samples contained 80-120 $\mathrm{A}_{260}$ O.D. units in 0.33 $\mathrm{ml}(0.9-1.4 \mathrm{mM})$. Isotope labeled sequence variants of RNA I (Figure S5) were prepared from $1.5 \mathrm{ml}$ transcriptions.

NMR Spectroscopy. Spectra were acquired on Varian Inova $500 \mathrm{MHz}\left({ }^{1} \mathrm{H}-\left[{ }^{13} \mathrm{C},{ }^{15} \mathrm{~N},{ }^{31} \mathrm{P}\right]\right.$ probe) and $600 \mathrm{MHz}\left({ }^{1} \mathrm{H}-\left[{ }^{13} \mathrm{C},{ }^{15} \mathrm{~N}\right]\right.$ cryoprobe $)$ spectrometers and were processed and analyzed using Felix 2007 (Felix NMR Inc., San Diego, CA). Two-dimensional (2D) ${ }^{13}$ C${ }^{1} \mathrm{H}$ HSQC spectra were collected to identify ${ }^{13} \mathrm{C}-{ }^{1} \mathrm{H}$ chemical shift correlations and to screen for RNA-compound interaction. Sugar spin systems were assigned using 3D $\mathrm{HCCH}-\mathrm{TOCSY}$ (8 ms and $24 \mathrm{~ms}$ DIPSI-3 spin lock) experiments collected in $\mathrm{D}_{2} \mathrm{O}$. Intraresidue base-sugar correlations were identified from H6-N1, H8-N9, and H1'-N1/N9 cross peaks in 2D H(C)N spectra. All pyrimidine correlations and several purine correlations were identified. Pyrimidine C2 and C4 resonances were assigned from H6- 
$\mathrm{C} 2$ and $\mathrm{H} 5-\mathrm{C} 4$ correlations using 2D H(CN)C and 2D CCH-COSY experiments ${ }^{21,22}$. 2D ${ }^{15} \mathrm{~N}-{ }^{1} \mathrm{H}$ HSQC spectra optimized for 2-bond HN couplings were collected to identify purine N7 and adenine N1 and N3 resonances. Sequential assignments and distance constraints for the non-exchangeable resonances were obtained at $26{ }^{\circ} \mathrm{C}$ from $2 \mathrm{D}{ }^{1} \mathrm{H}$ ${ }^{1} \mathrm{H}$ NOESY spectra $\left(t_{m}=120,160\right.$, and $\left.320 \mathrm{~ms}\right)$ and $3 \mathrm{D}{ }^{13} \mathrm{C}$-edited NOESY spectra $\left(\mathrm{t}_{\mathrm{m}}=\right.$ 160 and $320 \mathrm{~ms}$ ). Assignments and distance constraints for the exchangeable resonances were obtained at $15^{\circ} \mathrm{C}$ from $2 \mathrm{D}$ NOESY spectra $\left(\mathrm{t}_{\mathrm{m}}=160\right.$ and $\left.320 \mathrm{~ms}\right)$ acquired in $90 \%{ }^{1} \mathrm{H}_{2} \mathrm{O}$.

Distance and torsion angle constraints. Interproton distances were estimated from cross peak intensities in 2D NOESY and 3D ${ }^{13} \mathrm{C}$-edited NOESY spectra. The covalently fixed pyrimidine $\mathrm{H} 5-\mathrm{H} 6$ distance $(\approx 2.4 \AA)$ and the conformationally restricted sugar $\mathrm{H} 1$ '-H2' distance (2.8-3.0 $\AA$ ) give rise to the most intense peaks in the NOESY spectra and were used as a reference to assign upper bound distances to proton pairs based on the relative intensities of the corresponding cross peaks (qualitatively classified as strong, medium, weak, or very weak in the 120 ms and 160 ms mixing time spectra). None of the cross peaks are as intense as the pyrmidine $\mathrm{H} 5-\mathrm{H} 6$ peaks, thus upper bound distance constraints were set to $3.2,4.2,5.2$, or $6.2 \AA$. Cross peaks observed only at mixing time 320 ms were classified as extremely weak and given $7.2 \AA$ upper bound distance constraints to account for the possibility of spin diffusion. All distance constraints were given lower bounds of $1.8 \AA$. Only the intra-residue sugar-to-sugar constraints involving $\mathrm{H}^{\prime}$ ' and $\mathrm{H}^{\prime}$ " resonances included in the calculations are considered conformationally restrictive. Distance constraints involving exchangeable 
protons were estimated from 320 ms mixing time NOESY spectra and were classified as medium, weak, very weak, or extremely weak.

Watson-Crick base pairs were identified by observation of a significantly downfield shifted $\mathrm{NH}$ or $\mathrm{NH} 2$ proton resonance and the observation of strong $\mathrm{G}-\mathrm{C} \mathrm{NH}-$ $\mathrm{NH}_{2}$ or $\mathrm{A}-\mathrm{U} \mathrm{H} 2-\mathrm{NH}$ NOEs and by the chemical shifts of non-protonated base ${ }^{15} \mathrm{~N}$ and ${ }^{13} \mathrm{C}$ carbonyl resonances. Hydrogen bonds were introduced as distance restraints of 2.9 $\pm 0.3 \AA$ between donor and acceptor heavy atoms and $2.0 \pm 0.2 \AA$ between acceptor and hydrogen atoms. Ribose ring pucker and backbone dihedral constraints were derived from ${ }^{3} \mathrm{~J}_{\mathrm{HH}},{ }^{3} \mathrm{~J}_{\mathrm{HP}}$, and ${ }^{3} \mathrm{~J}_{\mathrm{CP}}$ couplings ${ }^{23}$. Residues with ${ }^{3} \mathrm{~J}_{\mathrm{H} 1^{\prime}-\mathrm{H} 2^{\prime}}<5 \mathrm{~Hz}$ and C3' resonances between $70-74$ ppm were constrained to $\mathrm{C}^{\prime}$-endo. Ribose rings with ${ }^{3} \mathrm{~J}_{\mathrm{H} 1}$ H2' 5 Hz and with C3' and C4' resonances between 74-76 and 84-86 ppm, respectively, were left unconstrained. The angle $\delta$ was constrained as $85^{\circ} \pm 30^{\circ}$ and $160^{\circ} \pm 30^{\circ}$ for C3'-endo and C2'-endo sugars respectively. For residues 1 to 6, 9-12, 17-20, and 2328, $\gamma$ was constrained to the gauche ${ }^{+}$conformation $\left(60 \pm 20^{\circ}\right)^{23} \cdot \gamma$ was left unconstrained for the internal loop residues. Dihedral angle restraints for the $\beta$ and $\varepsilon$ torsion angles were estimated from ${ }^{3} \mathrm{~J}_{\mathrm{P}-\mathrm{C} 2} / \mathrm{P}-\mathrm{C} 4{ }^{\prime}$ couplings measured in 2D CECT-HCP 24 spectra. For stem residues, $\beta$ was constrained to the trans conformation $\left(180 \pm 20^{\circ}\right)$ if ${ }^{3} \mathrm{JP}_{\mathrm{P}-\mathrm{C} 4}$ ' was $>5 \mathrm{~Hz}$. $\varepsilon$ was constrained to the trans conformation $\left(-150 \pm 20^{\circ}\right)$ for residues with ${ }^{3} \mathrm{~J}_{\mathrm{P}-\mathrm{C} 2^{\prime}}<5 \mathrm{~Hz}$ and ${ }^{3} \mathrm{~J}_{\mathrm{P}-\mathrm{C} 4^{\prime}}>5 \mathrm{~Hz}$. $\alpha$ and $\zeta$ were constrained to $-65 \pm 20^{\circ}$ for the stem residues 1 to $5,10-12,17-19$, and $24-28$. A down-field shifted ${ }^{31} \mathrm{P}$ resonance is associated with the trans conformation of $\alpha$ or $\zeta$ and because no such shift is observed for any of the ${ }^{31} \mathrm{P}$ resonances, $\alpha$ or $\zeta$ were loosely constrained to $\left(0 \pm 120^{\circ}\right)$ which excludes the trans conformation for all other residues. Although all base $6 / 8-1$ ' intra- 
residue NOE cross peak intensities support the anti conformation about the glycosidic bond, no dihedral angle constraints were used for the angle $\chi$.

${ }^{1} \mathrm{H}-{ }^{13} \mathrm{C}$ residual dipolar coupling constants (RDCs) were determined from the measured frequency difference between corresponding proton doublets in HSQC spectra acquired for isotropic and Pf1 phage-aligned samples. RDC values from base $\mathrm{CH}$ and ribose 1' $\mathrm{CH}$ vectors were obtained in this way. The axial and rhombic terms were determined within XPLOR-NIH using an extensive grid search ${ }^{25}$, and yielded values of $\mathrm{D}_{a H}=-18.677$ and $\mathrm{R}_{h H}=0.153$.

NMR Structure calculations and refinement. An initial set of structures was calculated using a shortened version of the simulated annealing protocol (described below). A list of all proton pairs in the calculated structures closer than $5.0 \AA$ (representing expected NOEs) was compared to the list of constraints. The NOESY spectra were then reexamined for predicted NOEs absent from the constraint list. In some cases, this allowed the unambiguous assignment of previously unidentified NOEs, but, in other cases, the predicted NOEs were obscured due to spectral overlap.

Structure refinement was carried out with simulated annealing and restrained molecular dynamics (rMD) calculations using XPLOR-NIH v2.44 with the RNA ff1 force field and potentials implemented 25,26 . Starting RNA coordinates were generated as a single stranded oligoribonucleotide with C3'-endo ring puckers for all ribose moieties using 3DNA ${ }^{27}$. The structure calculations were performed in two stages. Beginning with the energy minimized starting coordinates, 50 structures were generated using 20 ps of rMD or 10000 steps, whichever is less, at $1200 \mathrm{~K}$ with hydrogen bond, NOE-derived distance and base-pairing restraints. The system then was cooled to $25 \mathrm{~K}$ over $0.2 \mathrm{ps}$ of 
rMD or 10000 steps, whichever is less. During this stage, RDC constraints and repulsive van der Waals forces were introduced into the system and the SANI force constraint used for RDCs was gradually increased from $0.010 \mathrm{kcal} \mathrm{mol}^{-1} \mathrm{~Hz}^{-2}$ to 1.000 kcal mol-1 $\mathrm{Hz}^{-2}$. Other force constants used for the calculations were increased-from 2 kcal mol-1 $\AA^{-2}$ to $30 \mathrm{kcal} \mathrm{mol}^{-1} \AA^{-2}$ for the NOE and from $10 \mathrm{kcal} \mathrm{mol}^{-1} \mathrm{rad}^{-2}$ to $100 \mathrm{kcal}$ $\mathrm{mol}^{-1} \mathrm{rad}^{-2}$ for the dihedral angle constraints. Each structure was then minimized with constraints at the end of the rMD. Eight structures were selected for the final refinement. The criteria for final structure selection included lowest energies, fewest constraint violations, and fewest predicted unobserved NOEs ( ${ }^{1} \mathrm{H}$ pairs less than $3.5 \AA$ apart, but no corresponding cross peak in the NOE spectra). A second round of rMD was performed on these structures using protocols similar to those used in the first round of structure calculation. The major difference was the starting temperature of $300 \mathrm{~K}$ followed by cooling to $25 \mathrm{~K}$ over 28 ps of rMD. Eight refined structures for each model were analyzed using XPLOR-NIH and UCSF Chimera ${ }^{28}$.

RNA structure ensemble generation. Coordinates from the energy minimized average NMR structure were used as the starting coordinates for a molecular dynamics (MD) simulation with GROMACS 5.05. The RNA molecule was solvated in TIP3P water and neutralized with sodium ions. After energy minimization, temperature and pressure of the system were equilibrated for 100 ps before the production run which continued for 100 ns. Snapshots were saved every 2 ps for further analysis.

The structure ensemble was generated by comparing the population average calculated RDC values with measured RDC values ${ }^{29,30}$. This method minimizes the number of conformers that satisfy all time-averaged RDC data and identifies a set of 
structures that represents unique and dominant populations across the entire RNA structure landscape. The structure ensemble was obtained using an in-house protocol termed EnsembleGen. The code and the detailed instruction for execution are freely available for download at https://imdlab.mdanderson.org/ressd/ressd.php. Combined with Relax 4.0.3 (http://wiki.nmr-relax.com/Relax_4.0.3), EnsembleGen uses one or more sets of RDC data to guide selection of RNA conformers from the pool of MD snapshots.

Virtual screen for motif-selective compounds. In silico high-throughput screening was performed as reported previously ${ }^{31}$ to identify small molecules potentially capable of specifically binding the UU:GA motif. Briefly, the ChemBridge diverse set $(\sim 50,080$ compounds) and MayBridge ( 14,400 compounds) were screened using GOLD docking software with GOLD Fitness scoring function ${ }^{32}$. The top 10 poses for each compound were saved for rescoring using the rDock solv scoring function ${ }^{33}$ and the top 3 poses were selected. These top poses were re-scored again using IMDLScore scoring function ${ }^{31}$, and the top $10 \%$ compounds were selected for RNA binding studies.

Longer MD simulations were performed to elucidate the possible binding mechanism of a hit-RNA complex. The topology and charges of the small molecule were prepared using Gaussian09 at B3LYP/6-311++G(d,p) level of theory on the Texas Advanced Computing Center (TACC) cluster. The ff99bsc0 force field was used for the RNA and general AMBER force field (GAFF) for the hit prepared by ACPYPE ${ }^{34}$. The complex was solvated in TIP3P water and neutralized with sodium ions. The simulation boxes were prepared so that no RNA or small molecule atom was closer than $14 \AA$ to the edge. The system was minimized and equilibrated for 2 ns before the production run 
which was conducted for 660 ns. Snapshots were saved every 20 ps for further analysis.

\section{Results and Discussion}

Spectral assignments. The non-exchangeable ${ }^{1} \mathrm{H}$ and ${ }^{13} \mathrm{C}$ resonances of RNA I were assigned using standard heteronuclear methods ${ }^{35,36 .}$. Most of the base and ribose resonances are resolved at $298 \mathrm{~K}$ and $\mathrm{pH} 6.8$. None of the base or 1 ' resonances were observed to be exchange broadened which would be indicative of intermediate time scale motions. Sequential assignment of non-exchangeable resonances was accomplished using 2D NOESY and 3D ${ }^{13} \mathrm{C}$-edited NOESY spectra. The sequential NOE connectivities are continuous in the base -1 ' region $\left(\tau_{\mathrm{mix}}=160 \mathrm{~ms}\right)$ except at the

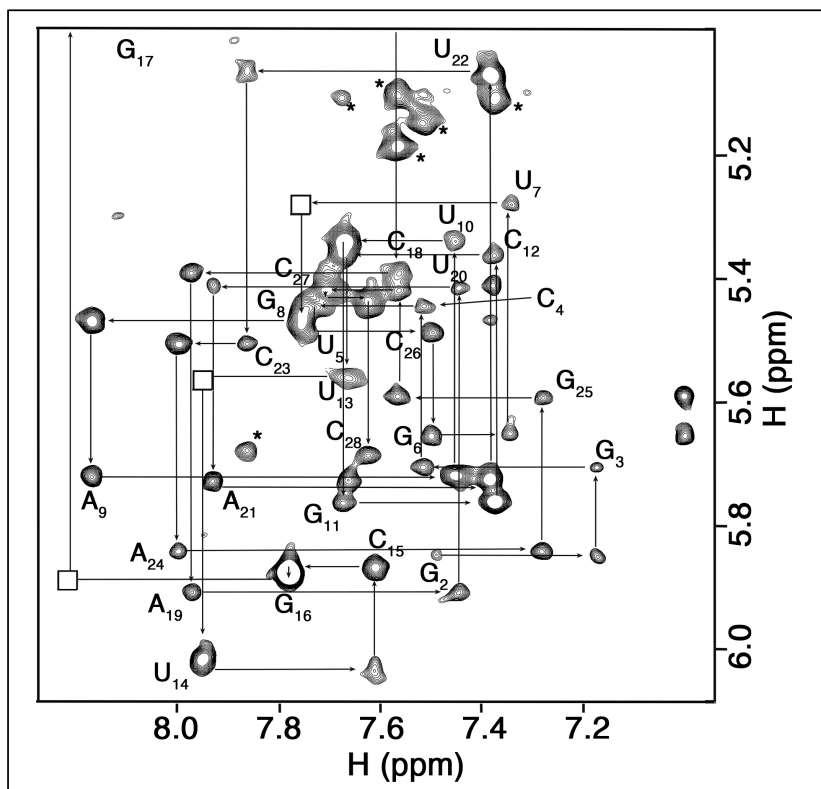

Figure 2. Base-1' region of a pyrimidine $\mathrm{C} 5$ filtered $2 D^{13} \mathrm{C}$ HSQC-NOESY spectrum ( $\mathrm{t}_{\mathrm{m}}=160 \mathrm{~ms}$ ) showing the sequential walk. Residual pyrimidine $\mathrm{H} 5-\mathrm{H} 6$ cross peaks are indicated by asterisks. The $\mathrm{U}_{7}-\mathrm{G}_{8}$ and $\mathrm{G}_{16}-\mathrm{G}_{17}$ inter-residue base- $\mathrm{H} 1$ ' cross peaks are weak, but present, in the corresponding 2D NOESY spectrum
$\mathrm{U}_{7}-\mathrm{G}_{8}$ step (Figure 2). The sequence specific resonance assignments are listed in Table S1.

The $\mathrm{NH}$ and cytidine $\mathrm{NH}_{2}$ resonances were assigned using ${ }^{1} \mathrm{H}-{ }^{1} \mathrm{H}$ NOESY and $\mathrm{HNCCH}$ experiments. The $\mathrm{NH}$ resonances of the stem base pairs, including the U-U base pair but not the G-A pair, yield NOE connectivities between neighboring base pairs. The $\mathrm{G}_{8}$ $\mathrm{NH}$ resonance was assigned based on ${ }^{1} \mathrm{H}$ and ${ }^{15} \mathrm{~N}$ chemical shifts. The inter- 
nucleotide phosphate ${ }^{31} \mathrm{P}$ resonances are clustered between -2.54 and $-4.60 \mathrm{ppm}$, but were not sequence specifically assigned.

NMR structure analysis. The solution structure of RNA I was calculated using a restrained molecular dynamics routine starting from 50 sets of randomized coordinates. The simulated annealing and refinement calculations were performed with 473 (305 NOE-derived and 68 hydrogen bond) conformationally restrictive distance constraints, 297 torsional angle constraints, and 51 RDC constraints (Table 1) leading to eight converged structures. The root mean square deviations (RMSDs) of the heavy atoms between the individual structures and the minimized mean structure is $0.194 \AA$.

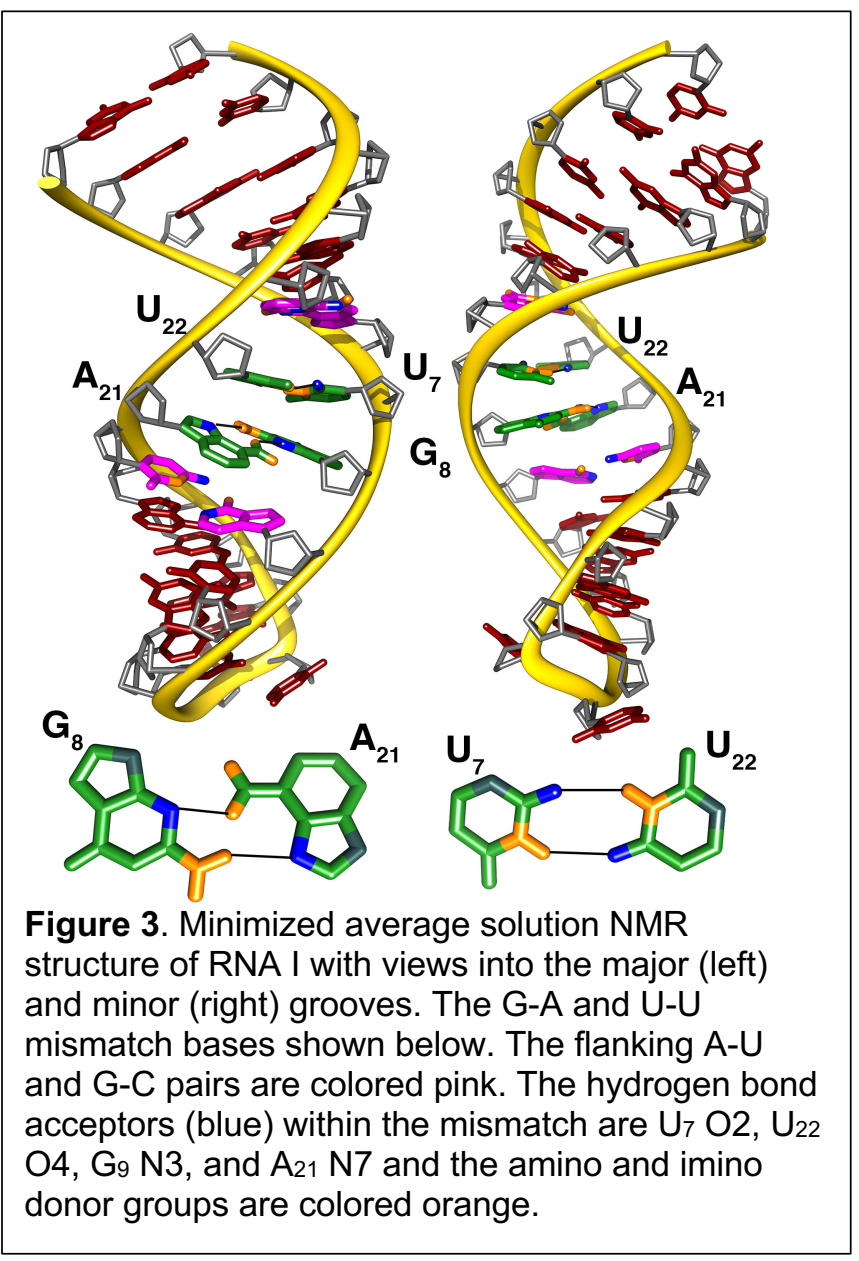

Structure statistics are summarized in

Table 1 and a representative structure is shown in Figure 3.

Structure around the UU:GA mismatch.

The primary interactions of interest within the mismatch region $\left(\mathrm{G}_{6}-\mathrm{A}_{9}\right.$ and $\mathrm{A}_{19-}$ $\mathrm{C}_{23}$ ) are those that define the base pairing pattern of nucleotides. $\mathrm{G}_{6}$ and $\mathrm{C}_{23}$ and $A_{9}$ and $U_{20}$ form Watson-Crick pairs, although the $\mathrm{U}_{20} \mathrm{NH}$ resonance displays solvent exchange broadening. The $U_{20}$ N3 chemical shift of $156 \mathrm{ppm}$ (identified through the $\mathrm{U}_{20} \mathrm{~N} 3-\mathrm{H} 5$ correlation) is consistent with an A-U base pair, but 
rapid exchange of the $U_{20} \mathrm{NH}$ resonance prevents confirmation of $A_{9}$ as the base pair partner. Notably, the $\mathrm{A}_{9} \mathrm{C} 2$ and $\mathrm{H} 2$ and $\mathrm{U}_{20}$ base 5 and 6 resonances do not display exchange broadening, pointing to increased solvent accessibility of the $\cup_{20}$ imino proton rather than increased dynamics of the $A_{9}$ or $U_{20}$ bases as the cause undetected $U_{20} \mathrm{NH}$ resonance. Consistent with the $\mathrm{H}(\mathrm{N}) \mathrm{CO}$ spectrum (Figure $\mathrm{S} 1$ ), $\mathrm{U}_{7}$ and $\mathrm{U}_{22}$ form an

Table 1. Summary of experimental distance and dihedral constraints and refinement statistics for RNA I.

\begin{tabular}{|c|c|c|}
\hline \multicolumn{2}{|l|}{ Constraint } & RNA I \\
\hline \multicolumn{2}{|c|}{ NOE distance constraints } & 305 \\
\hline \multicolumn{2}{|c|}{ Intra-residue ${ }^{a}$} & 144 \\
\hline \multicolumn{2}{|c|}{ Inter-residue } & 161 \\
\hline \multicolumn{2}{|c|}{ Mean number per residue } & 10.9 \\
\hline \multicolumn{3}{|c|}{ NOE constraints by category } \\
\hline Very strong & $(1.8-2.5 \AA)$ & 4 \\
\hline Strong & $(1.8-3.5 \AA)$ & 11 \\
\hline Medium & $(1.8-4.5 \AA)$ & 104 \\
\hline Weak & $(1.8-5.5 \AA)$ & 105 \\
\hline Very weak & $(1.8-6.5 \AA)$ & 71 \\
\hline \multicolumn{2}{|c|}{ H-bond distance constraints } & 33 \\
\hline \multicolumn{2}{|c|}{ Dihedral angle constraints } & 297 \\
\hline \multirow{2}{*}{\multicolumn{2}{|c|}{$\begin{array}{l}\text { Ribose ring }{ }^{b} \\
\text { Backbone }\end{array}$}} & 72 \\
\hline & & 157 \\
\hline \multicolumn{2}{|l|}{ Base pairs } & 68 \\
\hline \multicolumn{2}{|c|}{ Mean number per residue } & 10.6 \\
\hline \multicolumn{2}{|c|}{ RDC constraints } & 51 \\
\hline \multicolumn{2}{|l|}{ Violations } & 0 \\
\hline \multicolumn{2}{|c|}{ Average distance constraints $>0.3 \AA^{\mathrm{C}}$} & 0 \\
\hline \multirow{2}{*}{\multicolumn{2}{|c|}{$\begin{array}{l}\text { Average dihedral constraints }>0.5^{\circ} \mathrm{d} \\
\text { Average RDC constraints }>1.5 \mathrm{~Hz}\end{array}$}} & 0 \\
\hline & & 0 \\
\hline \multicolumn{3}{|c|}{ RMSD from average structure ${ }^{e}$} \\
\hline
\end{tabular}

aOnly conformationally restrictive constraints are included.

'Three torsion angles within each ribose ring were used to constrain the ring to either $\mathrm{C2}$ '-endo or C3'-endo conformation.

${ }^{\mathrm{c} A}$ distance violation of $0.3 \AA$ corresponds to $5.0 \mathrm{kcal}$ energy penalty.

${ }^{\mathrm{d} A}$ dihedral angle violation of $0.5^{\circ}$ corresponds to $0.05 \mathrm{kcal}$ energy penalty.

eCalculated against the minimized average structure. 
asymmetric base pair where the hydrogen bond acceptors are $\mathrm{O} 2$ and $\mathrm{O} 4$ carbonyl oxygens on $U_{7}$ and $U_{22}$, respectively. The upfield shift of the $U_{22} H_{1}$ ' is consistent with the side by side (sheared) orientation of the $\mathrm{G}_{8}$ and $\mathrm{A}_{21}$ bases (Figure 3). Two NOEs of particular interest are the atypical $\mathrm{A}_{21} \mathrm{H} 2-\mathrm{G}_{8} \mathrm{H} 1$ ' cross strand $\mathrm{NOE}$ and the unusually intense sequential $\mathrm{A}_{21} \mathrm{H} 2-\mathrm{U}_{22} \mathrm{H} 1$ ' NOE cross peak (Figure 2). These interactions are consistent with the base of $A_{21}$ stacked into the helix and is slid towards the minor groove away from the helix axis.

The sugar-phosphate backbone through the mismatch region of the stem has Aform geometry (Figure 3). The ribose puckers of $\mathrm{G}_{6}-\mathrm{A}_{9}$ and $\mathrm{A}_{19}-\mathrm{C}_{23}$ are uniformly $\mathrm{C3}^{\prime}-$ endo (as they are throughout the stem). The phosphate backbone torsional angles among $\mathrm{G}_{6}-\mathrm{A}_{9}$ and $\mathrm{A}_{19}-\mathrm{C}_{23}$ are largely within regular $\mathrm{A}$-form geometry except angles $\alpha$ and $\beta$ between $A_{21}$ and $U_{22}$. $\alpha$ varies between gauche ${ }^{+}$and gauche- (rather than canonical gauche-) and $\beta$ also tends to adopt either the gauche ${ }^{+}$or gauche ${ }^{-}$ conformation rather than the standard trans conformation. The ${ }^{31} \mathrm{P}$ resonances are centered within the main cluster around $-4.0 \mathrm{ppm}$, which is consistent with none of the $\alpha$ - or $\zeta$-torsional angles adopting the trans conformation.

The structure and dynamical properties of the of the tandem mismatch of U-U and G-A pairs have been examined in other contexts. In the context of G-C flanking sequences (Figure 1), the UU:GA internal loop appears ordered with intra-stem stacking of the uridine bases, inter-stem stacking of the adenine base and bulging of the $\mathrm{G}$, but lacks regular base pairing. The arrangement of the internal loop is consistent with the $\mathrm{NH}$ resonances of the $U$ bases that are significantly weakened by either solvent or chemical exchange ${ }^{17}$. In a case where the order of the tandem mismatch is reversed 
and flanked by $U-G$ and $A-U$ base pairs (Figure 1), the $G$ and A adopt the sheared conformation. However, the uridine bases are not hydrogen bonded generally oriented with their imino groups facing each other ${ }^{11}$. The uridine $\mathrm{NH}$ resonances are not present in the spectrum, consistent with the absence of hydrogen bonding. A third structure of the UU:GA mismatch involves the substitution of pseudouridine ( $\Psi$ ) for the $5^{\prime}-U\left(5^{\prime}-\Psi G\right)$. In this structure, with the mismatch flanked by C-G and U-A pairs (Figure 1), the $\Psi-U$ also does not form hydrogen bonds. However, the G-A adopt the less common iminoimino conformation ${ }^{12}$. Taken together, the structure of UU:GA motif is not only determined by the tandem pair of mismatched bases, but also by the identities of the flanking base pairs.

Selection of the three-dimensional structure ensemble of RNA I. RNA molecules exhibit a wide range of motional time scales and amplitudes. Computational methods to probe the conformational states of RNA molecules have been used to identify structures capable of binding small molecules ${ }^{29,37}$. Thus, to explore the dynamics of RNA I and to generate ensemble structures that RNA I might sample, unconstrained molecular dynamics simulations with explicit solvent and counterions were performed using the minimized average coordinates of the solution structure. No significant conformational changes were observed through 100 ns of simulation. The RMSD between the starting structure and 50,000 snapshots from the simulation trajectory is illustrated in Figure S2.

Although the RMSDs of the RNA I coordinates during the simulation do not reveal dramatic structure changes, examination of individual snapshots shows that localized conformational fluctuations occur throughout RNA I, particularly around the mismatched bases. To address the structural flexibility of RNA I and improve the 
potential for success of the small molecule virtual screen, a structure ensemble was created by incorporating RDCs into the structure selection process using our in-house program EnsembleGen. Figure S3 compares the calculated and measured RDC values, with overall RMSD $=4.12 \mathrm{~Hz}$. An ensemble of structures that reflects the unique and dominant conformations of nucleotides across the entire RNA structure landscape was constructed by determining the minimum number of RNA conformers that satisfy all time-averaged RDC data ${ }^{38}$. The all heavy atom RMSDs between the minimized average NMR structure and the individual structures contained in the ensemble range 1.65-5.03 $\AA$ (Figure S2). The representative structures of RNA I from the dynamic ensemble are shown in Figure S2.

Identification of small molecules specific for the UU:GA motif in RNA I. Previously, we examined computational docking tools to assess the predictive accuracy of experimental binding energies and established a protocol for RNA-small molecule docking ${ }^{31}$. For each of the 20 conformers in the RNA I structure ensemble, virtual screening was performed against 64,480 commercially available small molecules from the ChemBridge and MayBridge databases. Using GOLD and rDock in a sequential docking procedure, a total of 16 compounds (Table S2) were selected for experimental validation based on their docking scores (top 100), size (MW < 400), and chemical similarity (Tanimoto Coefficient $<0.6$ ). We also inspected the predicted binding poses for these compounds to ensure that the interactions between compounds and RNA are reasonable. Since no inhibitor has been reported as a reference, the criteria of reasonability is arbitrary, but we are especially interested in several features: docked sites (major groove vs. minor groove vs. intercalation between base pairs), interactions 
with based pairs vs. backbone, hydrogen bonding interactions, and aromatic stacking interactions.

NMR spectroscopy was used to evaluate the interaction of the 16 selected compounds with RNA I. Binding of a compound is expected to produce changes in the NH proton spectrum of RNA I. Although these compounds required the presence of 8-

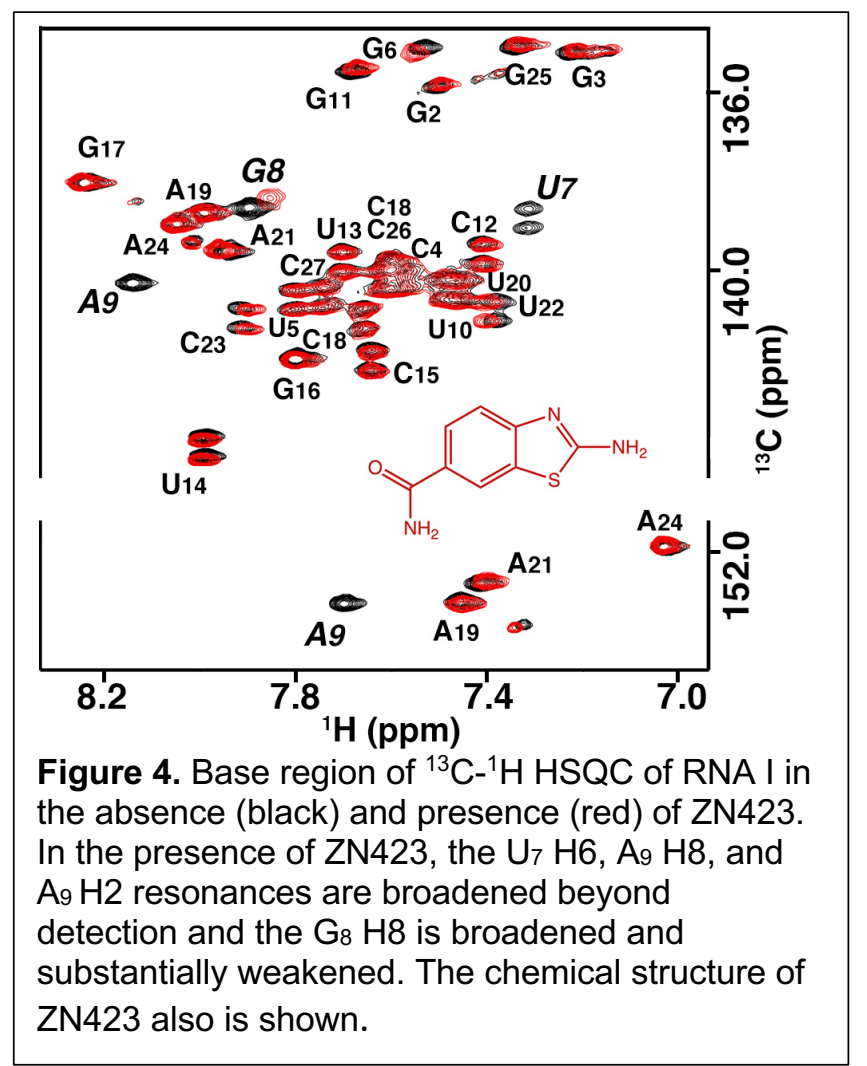
10\% DMSO for solubility, concentrations of DMSO up to $15 \%$ caused no perturbations to the $\mathrm{NH}$ and base ${ }^{13} \mathrm{C}-{ }^{1} \mathrm{H}$ spectra of RNA I. This observation is consistent with previous NMR spectral studies of RNA utilizing DMSO as a cosolvent ${ }^{39}$. Of the 16 compounds tested, two of them, 2-amino-1,3-benzothiazole6-carboxamide (ZN423) and 5,7dimethyl-1,4-dihydro-2,3quinoxalinedione (ZN449), were found to perturb the $\mathrm{NH}$ spectrum, with the former causing substantial changes to the $\mathrm{U}_{22}$ and $\mathrm{G}_{6}$ peaks (Figure S4). ZN423 also leads to changes in the non-exchangeable ${ }^{13} \mathrm{C}-{ }^{1} \mathrm{H}$ HSQC spectrum (Figure 4). The base 2 and 8 resonances of $A_{9}$ and base 6 resonance of $U_{7}$ are exchange broadened beyond detection by $Z N 423$ and the base 8 resonance of $G_{8}$ is significantly weakened. Also, the $G_{8}$ and $U_{20} 1$ ' are exchange broadened by ZN423 (Fig 4). 
To examine the selectivity of ZN423 for the UU:GA motif, RNA spectra of hairpins containing substitutions of nucleotides within and flanking the mismatch loop were screened in the presence of $\mathrm{ZN} 423$. The base $\mathrm{CH}$ spectrum of a $\mathrm{U}_{7} \mathrm{C}$ variant that introduces a $\mathrm{C}_{7}-\mathrm{U}_{22}$ mismatch displays the same exchange broadening pattern as the native molecule except that the $\mathrm{G}_{8}$ base resonance is completely exchange broadened in the $U_{7} C$ variant. $A U_{7} A$ variant displays no spectral changes in the presence of ZN423, but interestingly destabilizes the $A_{9}-U_{20}$ and $G_{8}-A_{21}$ pairs, as evidenced by exchange broadening of the $A_{9}, U_{20}$, and $A_{21}$ base resonances. Similarly, the spectra of $\mathrm{U}_{22} \mathrm{C}$ and $\mathrm{A}_{21} \mathrm{C}$ variants displayed no changes when $\mathrm{ZN} 423$ was added.

The effect of changing the flanking sequences was also examined. No evidence of binding could be observed after swapping the $A_{9}-U_{20}$ base pair with $G_{9}-C_{20}$ and $C_{9}-$ $G_{20}$. Similarly, binding is significantly diminished when the $U_{10}-A_{19}$ pair is replaced with $\mathrm{G}_{10}-\mathrm{C}_{19}$. The $\mathrm{U}_{5}$ was replaced with an adenine nucleotide to create an $A_{5}-A_{24}$ mismatch and decrease the stem stability without completely disrupting the $U_{7}-U_{22}$ pair. This modification also appears to decrease the binding (diminished resonance broadening) of compound ZN423. The results of the various substitutions on ZN423 interaction with the RNA are summarized in Figure S5.

Modeling of RNA-ligand structures. The binding affinity between ZN423 and RNA I is moderate $(>0.1 \mathrm{mM})$, resulting in chemical exchange on the intermediate time scale and preventing solution NMR structure determination of the complex. No NOEs between RNA I and ZN423 could be identified in long or short mixing time NOESY spectra. However, the H5 of ZN423 displays exchange broadening in the presence of RNA I whereas $\mathrm{H} 7$ and $\mathrm{H} 8$ appear unchanged, suggesting a more proximal relationship of one 
edge of ZN423 with RNA I (Figure S6). Thus, to better understand the interaction of

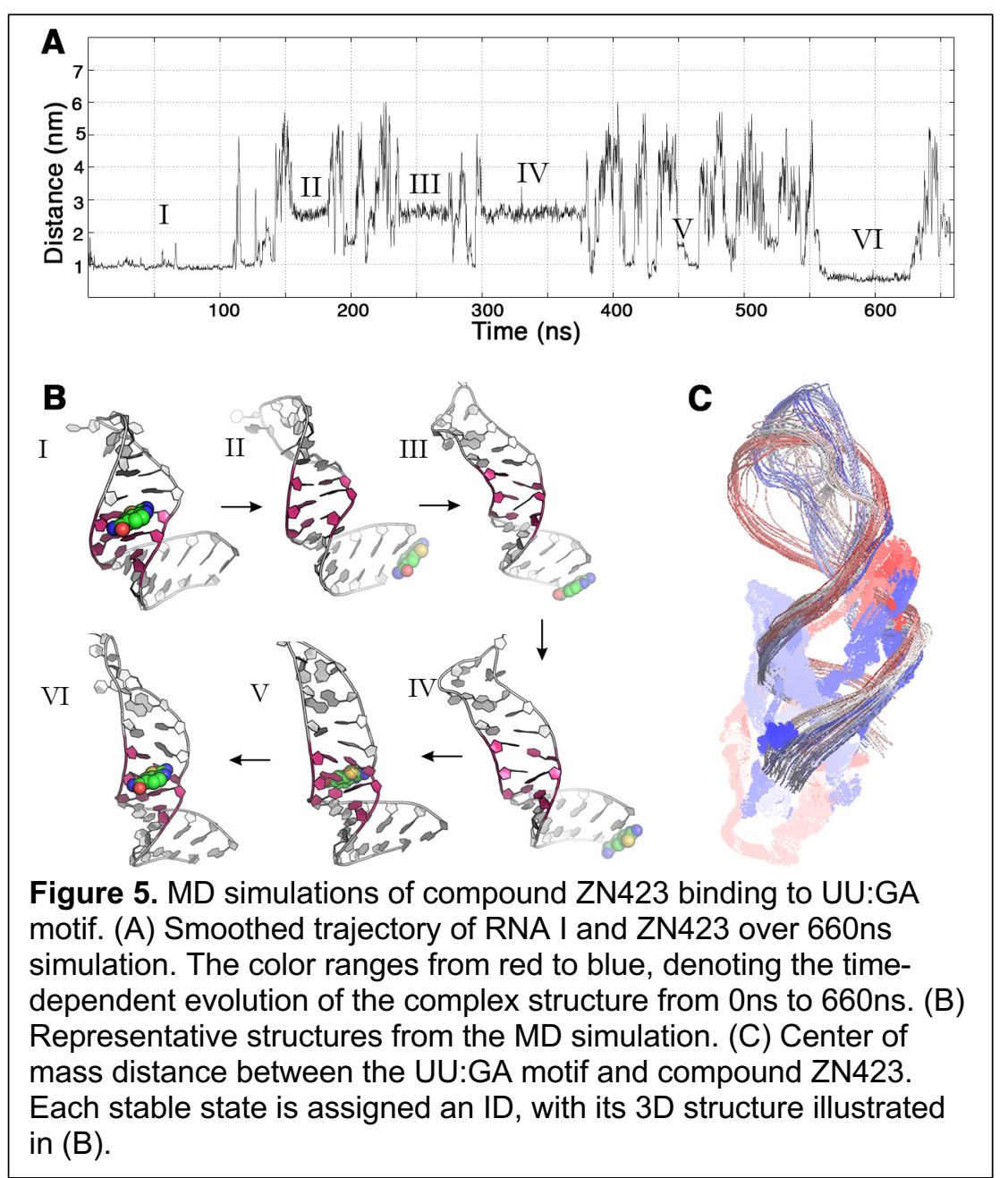

ZN423 with the UU:GA

internal loop,

unconstrained MD

simulations of RNA I with

ZN423 were performed.

Figure $5 \mathrm{~A}$ shows the

smoothed trajectory of

compound ZN423 over the

$\sim 660$ ns simulation in

explicit solvent.

Interestingly, we observed

a full binding cycle of

ZN423's association and

dissociation with the

UU:GA motif (Figure 5A-B) and identified six states in which ZN423 interacts with the

RNA (Figure 5B). The compound quickly bound the minor groove of RNA I at the

UU:GA motif, with a center of mass (COM) distance (Figure $5 \mathrm{C}$ ) of $10 \AA$ between UU:GA

and ZN423 by forming stable interactions (described below) for $\sim 100$ ns. The ZN423

molecule dissociated from RNA I and periodically (at $150 \mathrm{~ns}, 230 \mathrm{~ns}$, and $300 \mathrm{~ns}$ )

stacked against the terminal nucleotides. The compound also interacted with the major

groove in a non-specific and transient manner (at $\sim 450 \mathrm{~ns}$ ). Finally, it again bound to the

minor groove of the UU:GA motif. The two binding modes in the minor groove are nearly 
identical, with the latter having a COM distance of $8 \AA$. The residence time for the second minor groove binding event is $\sim 80 \mathrm{~ns}$, followed by a second binding cycle (Figure
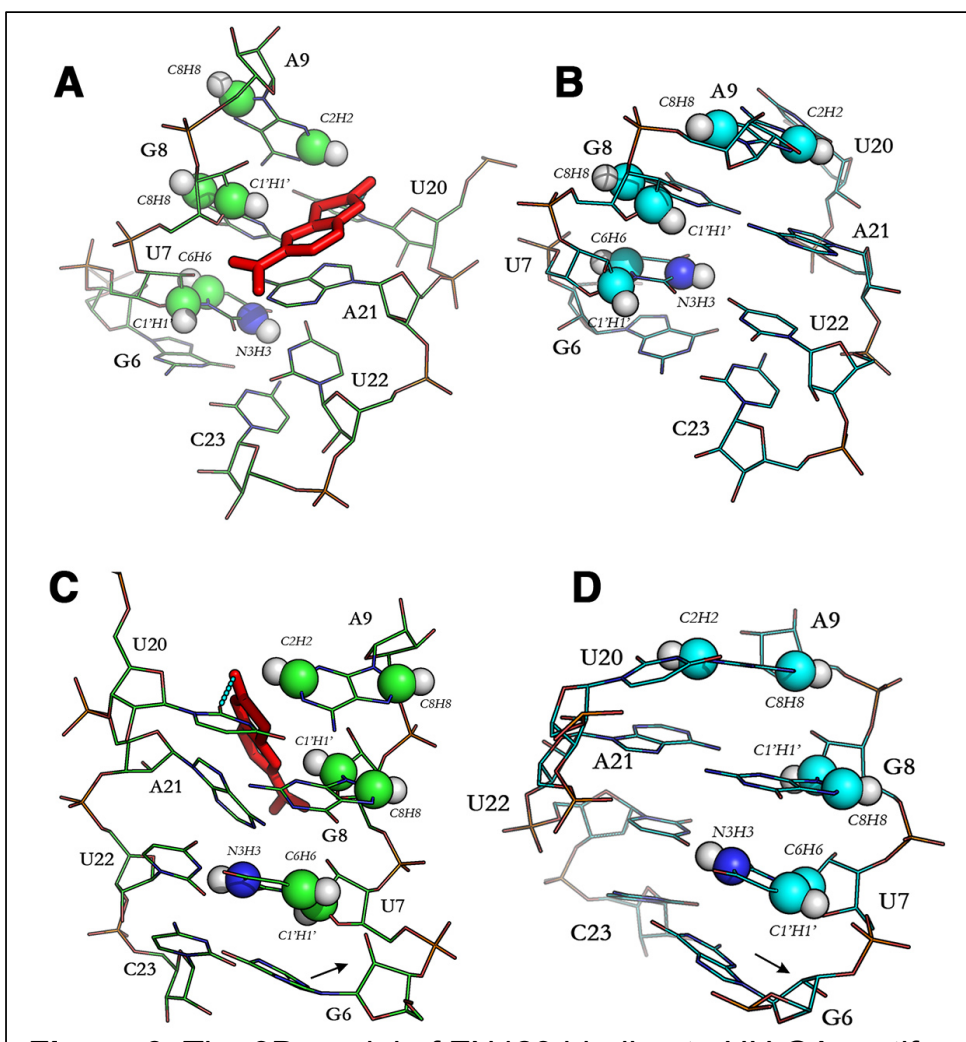

Figure 6. The 3D model of ZN423 binding to UU:GA motif. ZN423 is shown in red sticks, and the RNA atoms altered by the binding are shown in sphere. The changes of sugar puckering are highlighted with arrows. (A) Minor groove view of ZN423-bound complex structure. (B) Minor groove view of unbound RNA structure. (C) Major groove view of ZN423-bound complex structure. (D) Major groove view of unbound RNA structure.
$5 A)$.

\section{In both complexes}

involving the UU:GA motif,

ZN423 binds to the minor groove

defined by $G_{8}, A_{9}, U_{20}$, and $A_{21}$

(Figure 6A). The benzothiazole

moiety stacks on $A_{21}$, and the

amine group interacts with $U_{20}$

$\mathrm{O} 2$ to form an intermolecular $\mathrm{H}$ -

bond (Figure 6A and Figure 6C).

The sheared G-A base pair

exhibits a propeller twist of $40^{\circ}$

with the binding of ZN423 (Figure

6A). The moderate binding

affinity $(>0.1 \mathrm{mM})$ and the

positioning of ZN423 in the minor groove causes exchange broadening of several

resonances. The $\mathrm{A}_{9} \mathrm{H} 2$ and $\mathrm{G}_{8} \mathrm{H} 1$ ' are proximal to the amine and benzene $\mathrm{H} 5$,

respectively, of ZN423, which is consistent with the exchange broadening pattern. As

ZN423 binds, the propeller twist of the G-A pair increases significantly, altering the local

magnetic environments, and therefore chemical shifts, of several proximal protons

including $\mathrm{U}_{7} \mathrm{H}^{\prime}, \mathrm{U}_{22} \mathrm{H} 6, \mathrm{G}_{8} \mathrm{H} 8$ and $\mathrm{A}_{9} \mathrm{H} 8$. 
Moreover, as illustrated by Figure 7, destabilization of the two base pairs next to

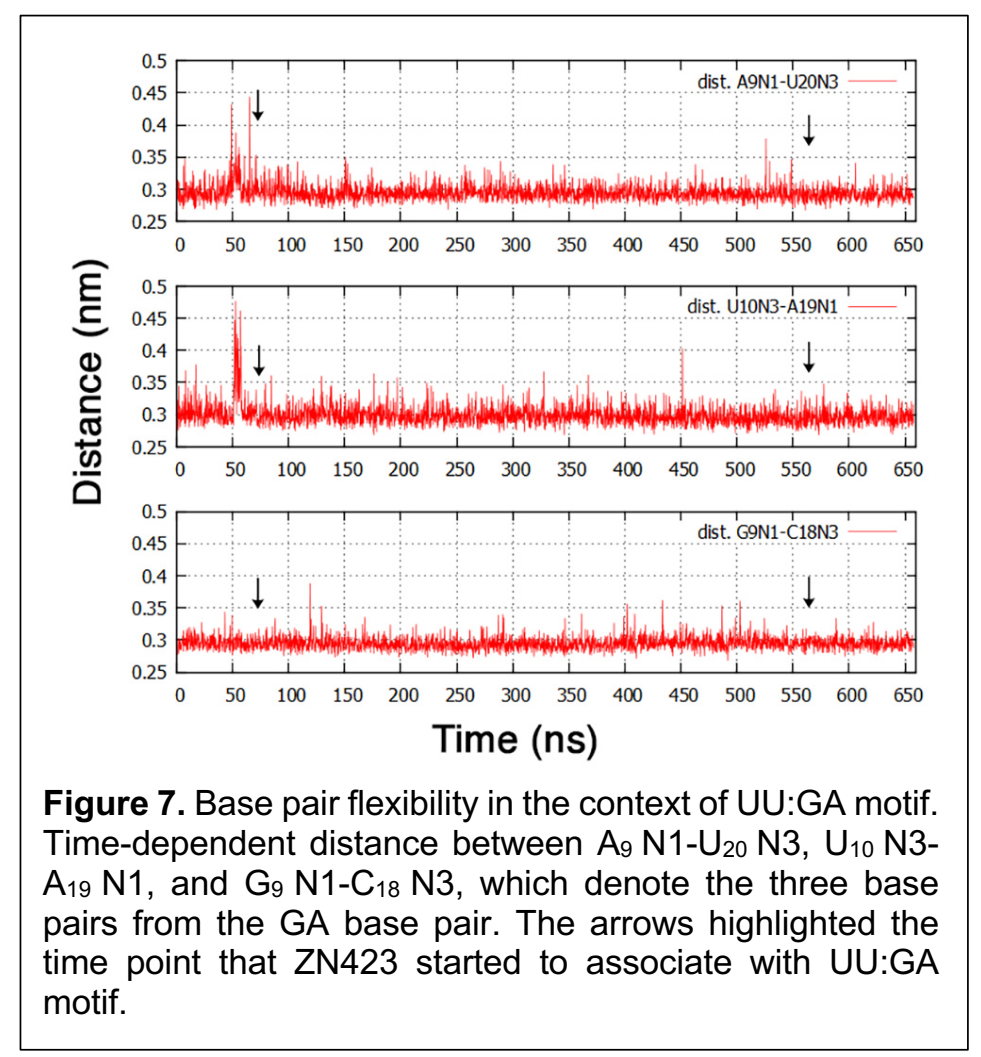
the GA pair is an essential step before ZN423 binds to the UU:GA motif. In comparison, the base pair stability at the +3 position does not correlate with the compound binding event. Thus, the MD simulation suggests the importance of flexibility on the GA side of the mismatch for binding affinity and this is consistent with the flanking sequence dependence on ZN423

binding observed by NMR (Figure S5).

We further assessed the AN423-RNA binding model indicated by the MD simulations experimentally using four derivatives of ZN423 (Table 2). The compounds were subjected to in silico docking analysis and titration with RNA I monitored by NMR. Any substitution at the R1, R2 or R3 positions failed to cause changes in the NMR spectra at concentration as high as $0.2 \mathrm{mM}$ (Table 2). This apparent lack of interaction is consistent with our structure models in which the amine group (R1) forms a hydrogen bond with $\mathrm{U}_{20}$ and the carboxamide group (R3) forms polar contacts with the $\mathrm{G}_{8}$ ribose ring such that any hydrophobic substitution would be expected to impair or abolish the 
binding. Removal of the carboxamide from R3 and substitution at R2 with a hydrazide moiety was also found to be detrimental to binding. Although the structure model

Table 2. Structure-activity relationship (SAR) of 423 series compounds

\begin{tabular}{|c|c|c|c|c|c|}
\hline Name & $\mathbf{R}_{1}$ & $\mathbf{R}_{\mathbf{2}}$ & $\mathbf{R}_{\mathbf{3}}$ & iMDLScore & Activity \\
\hline ZN423 & $-\mathrm{H}$ & $-\mathrm{H}$ & $-\mathrm{CONH}_{2}$ & -11.17 & active \\
\hline ZN423-1 & $-\mathrm{H}$ & $-\mathrm{H}$ & $-\mathrm{NHCOCH}_{3}$ & -9.99 & inactive \\
\hline ZN423-2 & $-\mathrm{H}$ & $-\mathrm{H}$ & $-\mathrm{CH}\left(\mathrm{CH}_{3}\right) \mathrm{CH}_{3}$ & -10.32 & inactive \\
\hline ZN423-3 & $-\mathrm{H}$ & $-\mathrm{CONHNH}{ }_{2}$ & $-\mathrm{H}$ & -6.64 & inactive \\
\hline ZN423-4 & $-\mathrm{NH}_{2}$ & $-\mathrm{H}$ & $-\mathrm{CONHNH}_{2}$ & -10.48 & inactive \\
\hline
\end{tabular}

suggests that R2 is exposed to the solvent and thus is expected to minimally contribute to binding, the hydrazide group cannot substitute for the simultaneous loss of the R3 carboxamide-RNA interaction. As shown in Table 2, evaluations with the RNA-specific scoring function iMDLScore ${ }^{31}$ indicate that ZN423 has the highest rank of binding compared with the four derivatives. This result is consistent with the inability of the derivatives to bind RNA I. Although the score difference itself is not significant for ZN423 and ZN423-4 (e.g., -11.17 vs. -10.48), the loss of activity for ZN423-4 is not surprising because of the possibility of the hydrazide group at R3 to form intra-molecular hydrogen bonds ${ }^{40}$ in addition to the extra size of the hydrazine moiety at R1 position.

\section{Conclusion}

RNAs are historically important therapeutic targets, but they have been less systematically investigated than proteins as targets. X-ray crystallographic and NMR structure studies of ribosomes and rRNA fragments revealed that various aminoglycoside antibiotics inhibit translation by direct binding to architectural elements within the 16S rRNA and demonstrated that RNA can be specifically targeted by small 
molecules. The array of RNA motifs that can be specifically bound by small molecules and the chemotypes of those small molecules has been expanded through small molecule library-RNA motif library screens ${ }^{1,41}$. However, with few exceptions ${ }^{30}$, rational design of chemical inhibitors targeting specific RNA motifs has been less productive due in part to lack of reliable in silico and experimental tools for structure-based drug design.

Previously, we evaluated the ability of computational docking to predict experimental binding energies between RNA and small molecules and established a protocol for docking of these complexes ${ }^{31}$. However, a significant challenge in accurately docking small molecules to RNA is that the structure of the complexed form of the target RNA is generally unknown. Al-Hashimi and colleagues demonstrated the improved accuracy of in silico docking predictions by using multiple conformers of the internal loop region of the TAR RNA hairpin derived from NMR-informed MD simulations 30. In this study we employed a similar method to screen chemical libraries for small molecule compounds with the potential to specifically bind an RNA containing a UU:GA tandem mismatch. This sequence motif has been predicted to be present in ncRNAs, such as immature miRNAs, implicated in many diseases including cancer. Binding studies with one of the hits identified in our screen, 2-amino-1,3-benzothiazole-6carboxamide (ZN423), revealed that the motif includes not only the tandem mismatch but also the flanking base pairs. These base pairs contribute to the structural and/or dynamic features necessary to support binding of ZN423. Although not wholly unexpected, the finding that specificity for the UU:GA mismatch is flanking sequence dependent demonstrates the importance of context effect and increases the possible number of small non-canonical features that can be specifically targeted. In addition, 
while the UU:GA tandem mismatch, or more generally YU:GA motif, may appear in multiple RNA molecules, the flanking sequence requirements increase the specificity of ZN423 for only a small subset of RNA molecules.

Finally, ZN423 is small even by small molecule standards and is more akin to a molecular fragment. Thus, the moderate binding affinity $(>0.1 \mathrm{mM})$ for the UU:GA mismatch may be expected to be optimized systematically based on the predicted binding pose within RNA I. The ZN423 series compounds available for testing demonstrates the importance of the carboxamide functionality at position R3, but are less informative of modifications that can be accommodated along the outward-facing opposite edge of ZN423.

\section{SUPPORTING INFORMATION}

Six figures, a table of chemical shifts, and a table listing the top 16 hit compounds are provided.

\section{ACCESSION NUMBERS}

Coordinates have been deposited in the Protein Data Bank under accession number

PBD ID: 6VZC. Chemical shifts have been deposited in the Biomolecular Magnetic

Resonance Bank under accession number BMRB ID: 50197.

\section{ACKNOWLEDGEMENTS}

We thank Malgorzata Michnicka for preparation of the T7 RNA polymerase and synthesis of the labeled 5'-nucleotide triphosphates.

\section{FUNDING}


This work was supported by National Science Foundation grants CHE-1412864 and

CHE-1411859 to E.P.N. and S.Z., respectively. S.Z. is also partially supported by

CPRIT RP170333 and MD Anderson IRG grant. The computation time was provided by

MD Anderson and UT Austin TACC HPC resources.

\section{REFERENCES}

[1] Tran, T., and Disney, M. D. (2011) Molecular recognition of 6'-N-5-hexynoate kanamycin A and RNA $1 \times 1$ internal loops containing CA mismatches, Biochemistry 50, 962-969.

[2] Haniff, H. S., Graves, A., and Disney, M. D. (2018) Selective Small Molecule Recognition of RNA Base Pairs, ACS Comb Sci 20, 482-491.

[3] Sztuba-Solinska, J., Shenoy, S. R., Gareiss, P., Krumpe, L. R., Le Grice, S. F., O'Keefe, B. R., and Schneekloth, J. S., Jr. (2014) Identification of biologically active, HIV TAR RNA-binding small molecules using small molecule microarrays, J Am Chem Soc 136, 8402-8410.

[4] Di Giorgio, A., and Duca, M. (2019) Synthetic small-molecule RNA ligands: future prospects as therapeutic agents, Medchemcomm 10, 1242-1255.

[5] Sztuba-Solinska, J., Chavez-Calvillo, G., and Cline, S. E. (2019) Unveiling the druggable RNA targets and small molecule therapeutics, Bioorg Med Chem 27, 2149-2165.

[6] Fulle, S., and Gohlke, H. (2010) Molecular recognition of RNA: challenges for modelling interactions and plasticity, J Mol Recognit 23, 220-231.

[7] Thomas, J. R., and Hergenrother, P. J. (2008) Targeting RNA with small molecules, Chemical reviews 108, 1171-1224.

[8] Disney, M. D., Velagapudi, S. P., Li, Y., Costales, M. G., and Childs-Disney, J. L. (2019) Identifying and validating small molecules interacting with RNA (SMIRNAs), Methods in enzymology 623, 45-66.

[9] Rizvi, N. F., Santa Maria, J. P., Jr., Nahvi, A., Klappenbach, J., Klein, D. J., Curran, P. J., Richards, M. P., Chamberlin, C., Saradjian, P., Burchard, J., Aguilar, R., Lee, J. T., Dandliker, P. J., Smith, G. F., Kutchukian, P., and Nickbarg, E. B. (2019) Targeting RNA with Small Molecules: Identification of Selective, RNABinding Small Molecules Occupying Drug-Like Chemical Space, SLAS Discov, 2472555219885373.

[10] Zhang, P., Park, H. J., Zhang, J., Junn, E., Andrews, R. J., Velagapudi, S. P., Abegg, D., Vishnu, K., Costales, M. G., Childs-Disney, J. L., Adibekian, A., Moss, W. N., Mouradian, M. M., and Disney, M. D. (2020) Translation of the intrinsically disordered protein alpha-synuclein is inhibited by a small molecule targeting its structured mRNA, Proc Natl Acad Sci U S A 117, 1457-1467.

[11] Jain, N., Morgan, C. E., Rife, B. D., Salemi, M., and Tolbert, B. S. (2016) Solution Structure of the HIV-1 Intron Splicing Silencer and Its Interactions with the UP1 
Domain of Heterogeneous Nuclear Ribonucleoprotein (hnRNP) A1, J Biol Chem 291, 2331-2344.

[12] Bilbille, Y., Vendeix, F. A., Guenther, R., Malkiewicz, A., Ariza, X., Vilarrasa, J., and Agris, P. F. (2009) The structure of the human tRNALys3 anticodon bound to the HIV genome is stabilized by modified nucleosides and adjacent mismatch base pairs, Nucleic Acids Res 37, 3342-3353.

[13] Davlieva, M., Donarski, J., Wang, J., Shamoo, Y., and Nikonowicz, E. P. (2014) Structure analysis of free and bound states of an RNA aptamer against ribosomal protein S8 from Bacillus anthracis, Nucleic Acids Res 42, 10795-10808.

[14] Seok, J. K., Lee, S. H., Kim, M. J., and Lee, Y. M. (2014) MicroRNA-382 induced by HIF-1alpha is an angiogenic miR targeting the tumor suppressor phosphatase and tensin homolog, Nucleic Acids Res 42, 8062-8072.

[15] Wang, H., Yan, B., Zhang, P., Liu, S., Li, Q., Yang, J., Yang, F., and Chen, E. (2020) MiR-496 promotes migration and epithelial-mesenchymal transition by targeting RASSF6 in colorectal cancer, J Cell Physiol 235, 1469-1479.

[16] Yan, T. T., Ren, L. L., Shen, C. Q., Wang, Z. H., Yu, Y. N., Liang, Q., Tang, J. Y., Chen, Y. X., Sun, D. F., Zgodzinski, W., Majewski, M., Radwan, P., Kryczek, I., Zhong, M., Chen, J., Liu, Q., Zou, W., Chen, H. Y., Hong, J., and Fang, J. Y. (2018) miR-508 Defines the Stem-like/Mesenchymal Subtype in Colorectal Cancer, Cancer Res 78, 1751-1765.

[17] Shankar, N., Xia, T., Kennedy, S. D., Krugh, T. R., Mathews, D. H., and Turner, D. $H$. (2007) NMR reveals the absence of hydrogen bonding in adjacent UU and AG mismatches in an isolated internal loop from ribosomal RNA, Biochemistry 46, 12665-12678.

[18] Davanloo, P., Rosenberg, A. H., Dunn, J. J., and Studier, F. W. (1984) Cloning and Expression of the Gene for Bacteriophage-T7 Rna-Polymerase, $P$ Natl Acad SciBiol 81, 2035-2039.

[19] Milligan, J. F., Groebe, D. R., Witherell, G. W., and Uhlenbeck, O. C. (1987) Oligoribonucleotide synthesis using T7 RNA Polymerase and Synthetic DNA Templates, Nucleic Acids Research 15, 8783-8789.

[20] Nikonowicz, E. P., Sirr, A., Legault, P., Jucker, F. M., Baer, L. M., and Pardi, A. (1992) Preparation of C-13 and N-15 Labeled RNAs for Heteronuclear Multidimensional NMR Studies, Nucleic Acids Research 20, 4507-4513.

[21] Denmon, A. P., Wang, J., and Nikonowicz, E. P. (2011) Conformation effects of base modification on the anticodon stem-loop of Bacillus subtilis tRNA(Tyr), $J$ Mol Biol 412, 285-303.

[22] Wang, J., and Nikonowicz, E. P. (2011) Solution structure of the K-turn and Specifier Loop domains from the Bacillus subtilis tyrS T-box leader RNA, J Mol Biol 408, 99-117.

[23] Varani, G., Aboul-ela, F., and Allain, F. H. T. (1996) NMR investigation of RNA structure, Progress in Nuclear Magnetic Resonance Spectroscopy 29, 51-127.

[24] Legault, P., Jucker, F. M., and Pardi, A. (1995) Improved measurement of ${ }^{13} \mathrm{C},{ }^{31} \mathrm{P} \mathrm{J}$ coupling constants in isotopically labeled RNA, FEBS Letters 362, 156-160.

[25] Schwieters, C. D., Kuszewski, J. J., Tjandra, N., and Clore, G. M. (2003) The XplorNIH NMR molecular structure determination package, J Magn Reson 160, 65-73. 
[26] Yamazaki, T., Muhandiram, R., and Kay, L. E. (1994) Nmr Experiments for the Measurement of Carbon Relaxation Properties in Highly Enriched, Uniformly C13,N-15-Labeled Proteins - Application to C-13(Alpha) Carbons, J Am Chem Soc 116, 8266-8278.

[27] Lu, X. J., and Olson, W. K. (2003) 3DNA: a software package for the analysis, rebuilding and visualization of three-dimensional nucleic acid structures, Nucleic Acids Res 31, 5108-5121.

[28] Pettersen, E. F., Goddard, T. D., Huang, C. C., Couch, G. S., Greenblatt, D. M., Meng, E. C., and Ferrin, T. E. (2004) UCSF Chimera--a visualization system for exploratory research and analysis, J Comput Chem 25, 1605-1612.

[29] Frank, A. T., Stelzer, A. C., Al-Hashimi, H. M., and Andricioaei, I. (2009) Constructing RNA dynamical ensembles by combining MD and motionally decoupled NMR RDCs: new insights into RNA dynamics and adaptive ligand recognition, Nucleic Acids Res 37, 3670-3679.

[30] Stelzer, A. C., Frank, A. T., Kratz, J. D., Swanson, M. D., Gonzalez-Hernandez, M. J., Lee, J., Andricioaei, I., Markovitz, D. M., and Al-Hashimi, H. M. (2011) Discovery of selective bioactive small molecules by targeting an RNA dynamic ensemble, Nat Chem Biol 7, 553-559.

[31] Chen, L., Calin, G. A., and Zhang, S. (2012) Novel insights of structure-based modeling for RNA-targeted drug discovery, J Chem Inf Model 52, 2741-2753.

[32] Verdonk, M. L., Cole, J. C., Hartshorn, M. J., Murray, C. W., and Taylor, R. D. (2003) Improved protein-ligand docking using GOLD, Proteins 52, 609-623.

[33] Morley, S. D., and Afshar, M. (2004) Validation of an empirical RNA-ligand scoring function for fast flexible docking using Ribodock, J Comput Aided Mol Des 18, 189-208.

[34] Sousa da Silva, A. W., and Vranken, W. F. (2012) ACPYPE - AnteChamber PYthon Parser interfacE, BMC Res Notes 5, 367.

[35] Dieckmann, T., and Feigon, J. (1997) Assignment methodology for larger RNA oligonucleotides: application to an ATP-binding RNA aptamer, Journal of biomolecular NMR 9, 259-272.

[36] Pardi, A. (1995) Multidimensional heteronuclear NMR experiments for structure determination of isotopically labeled RNA, Methods in enzymology 261, 350-380.

[37] Zhang, Q., Sun, X., Watt, E. D., and Al-Hashimi, H. M. (2006) Resolving the motional modes that code for RNA adaptation, Science 311, 653-656.

[38] Clore, G. M., and Schwieters, C. D. (2004) Amplitudes of protein backbone dynamics and correlated motions in a small alpha/beta protein: correspondence of dipolar coupling and heteronuclear relaxation measurements, Biochemistry 43, 10678-10691.

[39] Lee, J., Vogt, C. E., McBrairty, M., and Al-Hashimi, H. M. (2013) Influence of dimethylsulfoxide on RNA structure and ligand binding, Analytical chemistry 85, 9692-9698.

[40] Schonbaum, G. R. (1973) New complexes of peroxidases with hydroxamic acids, hydrazides, and amides, J Biol Chem 248, 502-511.

[41] Childs-Disney, J. L., Tran, T., Vummidi, B. R., Velagapudi, S. P., Haniff, H. S., Matsumoto, Y., Crynen, G., Southern, M. R., Biswas, A., Wang, Z. F., Tellinghuisen, T. L., and Disney, M. D. (2018) A Massively Parallel Selection of 
bioRxiv preprint doi: https://doi.org/10.1101/2020.06.11.146761; this version posted June 11, 2020. The copyright holder for this preprint (which was not certified by peer review) is the author/funder. All rights reserved. No reuse allowed without permission.

Small Molecule-RNA Motif Binding Partners Informs Design of an Antiviral from Sequence, Chem 4, 2384-2404. 